\title{
ISCR2 and IS26: Two Insertion Sequences Highly Dispersed among Acinetobacter spp. Clinical Strains
}

\begin{abstract}
The aim of this work is to study the dispersion of two ISs, ISCR2 and IS26, which are known to contribute in the acquisition of resistance determinants and genome plasticity, among a collection of hundred and seventy-four Acinetobacter spp. clinical isolates. PCR amplification reactions using total DNA were performed to search ISCR2, IS26, and different antibiotic resistance genes (tet(B), aphA6, sul2, floR, $d f r 9$ ) previously described in the genetic context of ISCR2.

Among A. baumannii, positive amplification for ISCR2 was obtained in $66 \%$ of the included isolates and most of them (93\%) were positive for IS26 amplification. The platform tet(B)::ISCR2 was found in $57 \%$ of the ISCR2 positive isolates and only 14 gave negative amplification for tet(B). In these isolates positive amplifications of genes that were previously described associated to ISCR2 -such as, aphA6, sul2, floR, and dfr9- were observed. When searching these ISs in the non-baumannii Acinetobacter studied-collection, two isolates were positive for ISCR2 and two isolates for IS26. No positive results were obtained for tet(B), but the presence of $s u l 2$, floR and $d f r 9$ was found. Our results exposed a great dispersion of ISCR2 as well as IS26 among Acinetobacter spp. clinical isolates reinforcing the idea that the ISs play a crucial role in the plasticity and evolution towards drug resistance in this genus.
\end{abstract}

Keywords: Acinetobacter spp.; ISCR2; IS26; Insertion sequences; Minimum inhibitory concentration

\section{Short Communication}

Volume 4 Issue 1 - 2017

Montaña $\mathbf{S}^{1}$, Almuzara $\mathbf{M}^{2}$, Pennini $\mathbf{M}^{3}$, Sucari $A^{3}$, Centrón $D^{1}$, Vay $C^{2}$ and María Soledad Ramírez ${ }^{4 *}$

${ }^{1}$ Instituto de Microbiología y Parasitología Médica, Argentina ${ }^{2}$ Laboratorio de Bacteriología, Argentina

${ }^{3}$ Unidad Microbiología, Argentina

${ }^{4}$ Department of Biological Science, California State University Fullerton, USA

*Corresponding author: María Soledad Ramírez, Assistant Professor, Department of Biological Science

California State University Fullerton, Fullerton, CA, USA, Email: msramirez@fullerton.edu

Received: December 26, 2016 | Published: January 26, 2017

\section{Introduction}

Acinetobacter are gram-negative, coccobacilli, non-fermenting aerobes and can cause a variety of nosocomial infections [14]. Currently forty-one distinctive Acinetobacter species with unique names and characteristics were described [3, (http:// www.bacterio.net/a/acinetobacter.html)]. Among this genus, $A$ baumannii is responsible for the majority of nosocomial infections and has an intrinsic ability to acquire and to develop antibiotic resistance determinants to all available antibiotics to treat it [5]. Patient outcomes associated with extensively drug-resistant $A$. baumannii infections can be deadly. The CDC reports 500 deaths annually due to infections with multidrug-resistant Acinetobacter (CDC). Recently due to advances in technologies used to identify bacterial species, other members of the Acinetobacter genus have been recovered in the clinical setting and have demonstrated resistance to different antibiotics $[3,6]$.

Insertion sequences (ISs) are important for the acquisition and dissemination of antimicrobial resistance determinants, and can also contribute to resistance by insertional inactivation of proteins, such as transcriptional regulators or outer membrane protein [6,7]. More than 30 different types of ISs have been reported in Acinetobacter spp. [8] supporting the idea that ISs play a crucial role in the evolution of this genus and that they have contributed to the development of the multidrug-resistant phenotype observed in this genus.

Among the ISs described in A. baumannii, most of the studies referred to ISAba1, ISAba125 and ISAba3 since they are associated with $b l a_{\text {OXA-like }}$ carbapenemases [7]. Among other ISs that have been not deeply studied, we found ISCR2. ISCR are recognized as powerful elements that can capture and mobilize antibiotic resistance genes, as well as, array extended clusters of antibiotic resistance genes on plasmids or on chromosomes [9]. Twenty-one members of the ISCR family have been described [10] and most of them are related with antibiotic resistance [11]. ISCR2 constitutes the second group of ISCR elements and it has been found in many species such as Vibrio cholerae, Shigella flexneri, Salmonella enterica, Escherichia coli, Klebsiella pneumoniae, Aeromonas salmonicida, Pasteurella piscicida. ISCR2 have been described on numerous plasmids carrying trimethoprim, tetracycline, chloramphenicol and sulphonamides resistance gene $[9,12]$ and also within resistance island [13-17].

Another important IS is IS26, which was recognized to play an important role in the acquisition and dissemination of genes that confer resistance to many different classes of antibiotics. It has been reported that IS26 plays a relevant role in the genomic plasticity observed in A. baumannii. They are involved in building transposons carrying different resistance genes in the fusion of additional transposons into resistance regions and also as a contributor of the variability observed among the genomic resistance island [18].

While conducting a surveillance exploring the dispersion of tetracycline resistance determinants among extensively-drug resistance (XDR) A. baumannii strains, we found a high prevalence of the genetic platform tet(B)::ISCR2 among minocycline resistant strains [19]. ISCR2 has been also described in Acinetobacter 
spp. associated with $b l a_{\mathrm{VEB} 1}$, suggesting its involvement in the acquisition and the mobilization of a $\beta$-lactamase [1]. Moreover, it was found as part of transposon and in AbaR-like resistant island close to tetracycline and aminoglycosides resistance genes [13-15]. The aim of the present work was to further explore the dispersion of this particular insertion sequence (ISCR2) among a collection of hundred and seventy-five XDR Acinetobacter spp. clinical isolates in order to expose its prevalence and its potential role as an important IS among XDR isolates. Moreover, we also explore the presence IS26 since is known to have an important role in the plasticity of this species.

A total of 174 Acinetobacter spp. isolates were recovered from a variety of clinical sites and samples from individual patients including blood, urine, and respiratory tract, among others, from 12 different hospitals during 2010-2014. One hundred and sixtyfour were $A$. baumannii, while the remaining ten were a novel species, Acinetobacter spp. A47 strain [2], A. pitti, A. radioresistans, A. johnsonii, A. ursingii, A. guilloiae. A. lwoffi, A. haemolyticus, A. soli and A. junii.

The species of the isolates were confirmed by MALDI-TOF MS (Bruker Daltonik, Bremen, Germany) and rpoB amplification and sequencing. The resistance profiles of the isolates to ampicilin, ampicilin/sulbactam, piperacillin/tazobactam, cefalotin, cefoxitin, cefotaxime, ceftazidime, cefepime, imipenem, meropenem, amikacin, gentamicin, nalidixic acid, ciprofloxacin, nitrofuratoin, colistin and trimetroprim/sulfamethoxazole were determined by disk diffusion according to Clinical Laboratory Standards Institute (CLSI) guidelines or using the VITEK 2 System (bioMerieux, Marcy, L'Etoile, France) employing the panel AST-082 (GNS susceptibility card) and the minimum inhibitory concentration (MIC) results were interpreted using the CLSI categories.

We extracted total DNA and used it to perform PCR amplification reactions according to the manufacturer's instructions (Promega, Madison, Wisconsin). Specific primers for ISCR2 (ISCR2F: AAGAATTTCTCCAATGCGGG and ISCR2R: GCGGCTCCTTTTCCGACAAC) and IS26 (IS26F: GCTGGCTGAACGCGGAG and IS26R: ATACCTTTGATGGTGGC) were used. Moreover, we searched different antibiotic resistance genes (tet(B), aphA6, sul2, floR, $d f r 9$ ) previously described in the genetic context of ISCR2 and we confirm its association in the required cases [9].

To confirm the association of antibiotic resistance genes (tet(B), aphA6, sul2, floR, dfr9) with ISCR2 several PCR products were sequenced after purifying the DNA by using the Wizard SV Gel and PCR clean-up System kit according to the manufacturer's directions (Promega, USA). Sequencing was performed on both DNA strands, using an ABI Prism 3100 Bio Analyzer equipment. The nucleotide sequences were analyzed using the Blast V2.0 software (http://www.ncbi.nlm.nih.gov/BLAST/).

\section{Conclusion}

All A. baumannii isolates were categorized as extreme drugresistant (XDR) according to Magiorakos et al. [20] being resistant to carbapenems and all antibiotics tested except colistin, and in some cases minocycline. Among non-baumannii Acinetobacter isolates, six were categorized as multi drug-resistant (MDR), and three were susceptible to all antibiotics tested (Table 1).

Table 1: Minimum inhibitory concentrations (MICs) of antimicrobial agents in non-baumannii Acinetobacter isolates.

\begin{tabular}{|c|c|c|c|c|c|c|c|c|c|c|c|c|c|c|c|c|c|}
\hline \multirow{2}{*}{ Isolate } & \multicolumn{17}{|c|}{$\operatorname{MIC}(\mathrm{mg} / \mathrm{L})^{\circ}$} \\
\hline & AMP & SAM & TZP & CEP & FOX & CTX & CAZ & CEF & IPM & MEM & AMK & GEN & NAL & CIP & NIT & COL & SXT \\
\hline 47 & $\leq 2$ & $\leq 2$ & $\leq 4$ & $\geq 64$ & $\leq 4$ & $\leq 1$ & $\leq 1$ & $\leq 1$ & $\leq 1$ & $\leq 0.25$ & $\leq 2$ & $\leq 1$ & 4 & $\leq 0.25$ & $\geq 512$ & $\leq 0.5$ & $\leq 20$ \\
\hline 23 & $\geq 32$ & $\leq 2$ & 16 & $\geq 64$ & ND & 8 & 4 & 2 & 4 & $\geq 16$ & 16 & $\leq 1$ & $\leq 2$ & $\leq 0.25$ & $\geq 512$ & $\leq 0.5$ & 160 \\
\hline 350 & 16 & $\leq 2$ & $\geq 128$ & $\geq 64$ & ND & 32 & $\geq 64$ & 8 & $\leq 0.25$ & 0.5 & $\leq 2$ & $\leq 1$ & 16 & $\leq 0.25$ & $\geq 512$ & $\leq 0.5$ & $\leq 20$ \\
\hline 306 & $\leq 2$ & $\leq 2$ & $\leq 4$ & $\leq 2$ & $\leq 4$ & $\leq 1$ & $\leq 1$ & $\leq 1$ & $\leq 1$ & $\leq 0.25$ & $\leq 2$ & $\leq 1$ & 8 & $\leq 0.25$ & 32 & 2 & $\leq 20$ \\
\hline 7 & 8 & $\leq 2$ & 8 & $\geq 64$ & ND & 8 & 8 & 2 & $\leq 0.25$ & $\leq 0.25$ & $\leq 2$ & $\leq 1$ & 4 & $\leq 0.25$ & 256 & $\leq 0.5$ & $\leq 20$ \\
\hline 181 & $>16$ & $>16 / 8$ & $>64 / 4$ & ND & $>16$ & ND & $>16$ & $>16$ & $>8$ & $>8$ & $\leq 8$ & $\leq 2$ & $\leq 8$ & $\leq 0.125$ & $>128$ & $\leq 1$ & $1 / 19$ \\
\hline 407 & $>16$ & $\leq 4 / 2$ & $\leq 4 / 4$ & ND & $>16$ & ND & 16 & 2 & $\leq 1$ & $\leq 1$ & $\leq 8$ & $\leq 2$ & $\leq 8$ & 0.5 & $>128$ & $\leq 1$ & $\leq 0.5 / 9.5$ \\
\hline 476 & $\leq 4$ & $\leq 4 / 2$ & $\leq 4 / 4$ & ND & $>16$ & ND & 4 & 4 & $\leq 1$ & $\leq 1$ & $\leq 8$ & $\leq 2$ & $\leq 8$ & $\leq 0.125$ & $>128$ & $\leq 1$ & $>2 / 38$ \\
\hline
\end{tabular}

${ }^{\circ}$ Bold indicates resistance.

AMP: Ampicilin; SAM: Ampicilin/Sulbactam; TZP: Piperacillin/Tazobactam; CEP: Cefalotin; FOX: Cefoxitin; CTX: Cefotaxime; CAZ: Ceftazidime; CEF: Cefepime; IPM: Imipenem; MEM: Meropenem; AMK: Amikacin; GEN: Gentamicin; NAL: Nalidixic Acid; CIP: Ciprofloxacin; NIT: Nitrofuratoin; COL: Colistin; SXT: trimetroprim/sulfamethoxazole; ND, no determinated. 
Surprisingly, in the A. baumannii isolates we obtained positive results for the amplification of ISCR2 in 108 out of 164 (66\%). The distribution of IS26 was also high among our isolates since 93\% $(153 / 164)$ of the isolates resulted positive for the amplification of this IS. This last result confirms the previous suggested role of IS26 as a ubiquitous and highly disperse IS [21]. Among the 164 A. baumannii isolates included in this study 102 of the isolates were harboring both IS. As we previously found a high association of ISCR2 with tet(B) in our A. baumannii population, we decided to investigate the presence of the previously reported platform tet(B)::ISCR2 [19]. We obtained positive results for the association of tet(B) with the ISCR2 element in $94 \mathrm{~A}$. baumannii isolates (Figure 1). Moreover, 14 of the isolates that were positive for ISCR2 and IS26 gave negative amplification for tet(B). In these 14 isolates we investigated for the presence of other genes (aphA6, sul2, floR, $d f r 9$ ) that were previously described associated to ISCR2. We found that all this isolates were positive for sul2, and in most $(13 / 14)$ of the cases other genes were also present. We found that four isolates were positive for sul2 and $d f r 9$, five were positive for floR apart of sul2, one was positive for aphA6, sul2 and $d f r 9$ and another was positive for floR, sul2 and $d$ fr9 (Figure 1). We were able to determine the association of ISCR2 with genes different than tet(B) only in one isolate, we were able to link ISCR2 with floR. Moreover, among ISCR2 negative isolates, we found three tet(B) positive isolates that were also IS26 negative.

On the other hand, in the non-baumannii Acientobacter isolates we obtained positives results for ISCR2 in two isolates, one of which was positive for sul2 and IS26 while the other was positive for sul2 and floR. Positives results for IS26 were found in two isolates, for sul2 in five isolates, for aphA6 in two isolates, and for floR in 1 isolate. No positive results were found for tet(B).

This study shows a great dispersion of ISCR2 as well as IS26 among Acinetobacter spp. clinical isolates reinforcing the idea that ISs plays a crucial role in the plasticity and evolution towards drug resistance in this genus. The association of ISCR 2 with tet(B) is the predominant platform among the $A$. baumannii isolates studied. However, ISCR2 is also present in other Acinetobacter spp. isolates where tet(B) is not present. We plan to perform further studies to find out the genetic environment of the ISCR2 positive isolates where tet(B) is not present. The high prevalence of ISCR2 and IS26 in our Acinetobacter spp. isolates allow us to suggest that this element is successfully been maintained is this species, giving a tool that could be involved in the acquisition and dissemination of resistance traits.

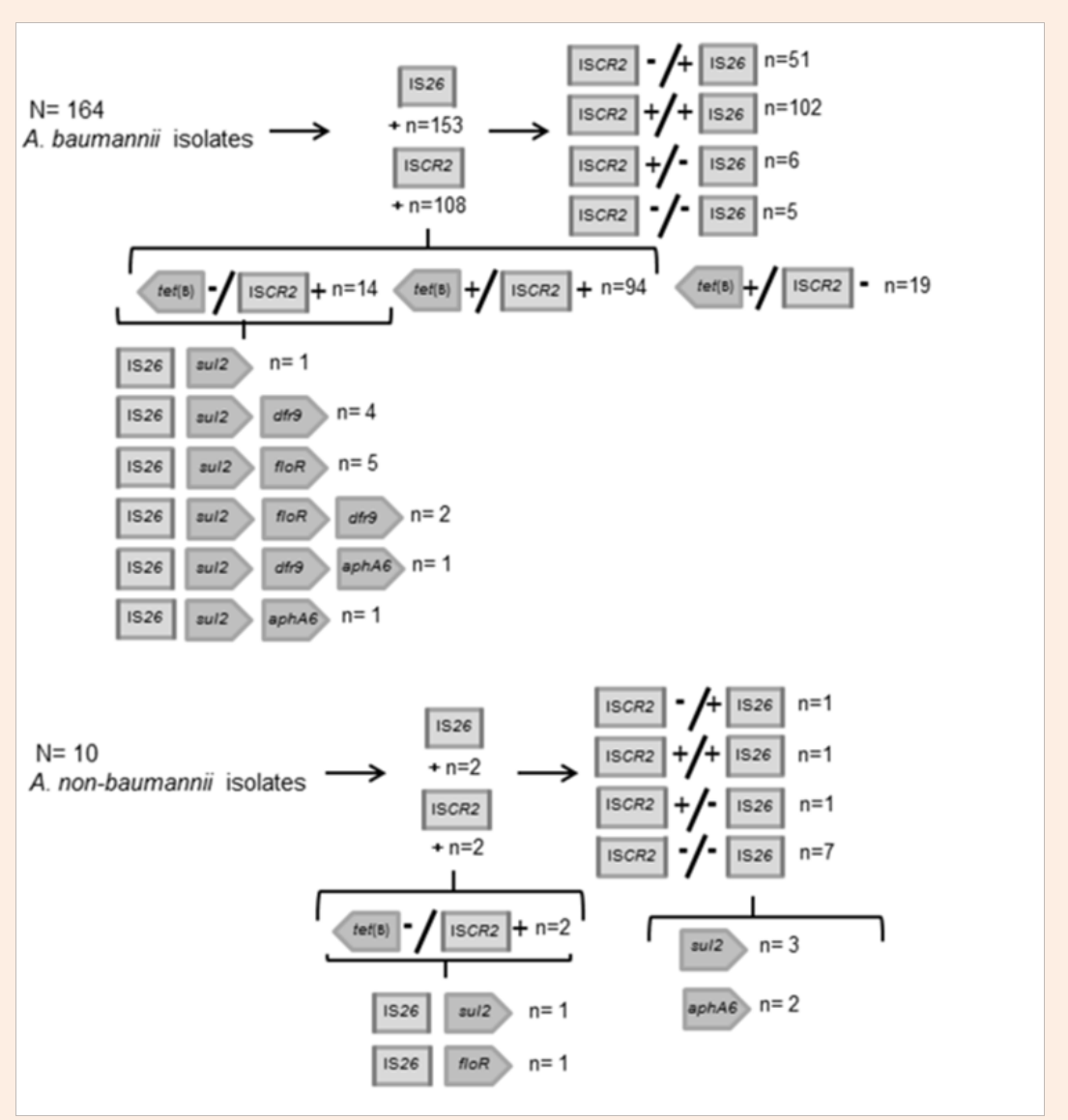

Figure 1: Diagram showing the dispersion of ISCR2, IS26 and the most prevalent genes associated to ISCR2 among A. baumannii and A. non baumannii isolates. 


\section{Acknowledgment}

M.S.R. and D.C are members of the career investigator of CONICET, Argentina. S.M. has a Doctoral Fellowship from CONICET. This study was supported by grants UBACyT and PICT 0120 to MSR, Buenos Aires, Argentina.

\section{References}

1. Poirel L, Mugnier PD, Toleman MA, Walsh TR, Rapoport MJ, et al. (2009) ISCR2, another vehicle for bla (VEB) gene acquisition. Antimicrob Agents Chemother 53(11): 4940-4943.

2. Almuzara M, Traglia GM, Krizova L, Barberis C, Montaña S, et al. (2015) A taxonomically unique Acinetobacter strain with proteolytic and hemolytic activities recovered from a patient with a soft tissue injury. J Clin Microbiol 53(1): 349-351.

3. Touchon M, Cury J, Yoon EJ, Krizova L, Cerqueira GC, et al. (2014) The genomic diversification of the whole Acinetobacter genus: origins, mechanisms, and consequences. Genome Biol Evol 8(11): 2866-2882.

4. Turton JF, Shah J, Ozongwu C, Pike R (2010) Incidence of Acinetobacter species other than A. baumannii among clinical isolates of Acinetobacter: evidence for emerging species. J Clin Microbiol 48(4): 1445-1449.

5. Peleg AY, de Breij A, Adams MD, Cerqueira GM, Mocali S, et al. (2012) The success of Acinetobacter species; genetic, metabolic and virulence attributes. PLoS One 7(10): e46984.

6. Roca I, Espinal P, Vila-Farrés X, Vila I (2012) The Acinetobacter baumannii oxymoron: commensal hospital dweller turned pan-drugresistant menace. Front Microbiol 3: 148.

7. Mussi MA, Limansky AS, Viale AM (2005) Acquisition of resistance to carbapenems in multidrug-resistant clinical strains of Acinetobacter baumannii: natural insertional inactivation of a gene encoding a member of a novel family of beta-barrel outer membrane proteins. Antimicrob Agents Chemother 49(4): 1432-1440.

8. Siguier P, Perochon J, Lestrade L, Mahillon J, Chandler M (2006) IS finder: the reference centre for bacterial insertion sequences. Nucleic Acids Res 34(Database issue): D32-D36.

9. Toleman MA, Bennett PM, Walsh TR (2006) ISCR Elements: novel gene-capturing systems of the $21^{\text {st }}$ century? Microbiol Mol Biol Rev 70(2): 296-316.

10. Schleinitz KM, Vallaeys $T$, Kleinsteuber S (2010) Structura characterization of ISCR8, ISCR22, and ISCR23, Subgroups of IS91Like Insertion Elements. Antimicrob Agents Chemother 54(10): 43214328.
11. Boerlin P, Reid Smith RJ (2008) Antimicrobial resistance: its emergence and transmission. Anim Health Res Rev 9(2): 115-126.

12. Toleman MA, Bennett PM, Walsh TR (2006) Common regions e.g. orf513 and antibiotic resistance: IS91-like elements evolving complex class 1 integrons. J Antimicrob Chemother 58(1): 1-6.

13. Nigro SJ, Post V, Hall RM (2011) The multiresistant Acinetobacter baumannii European clone I type strain RUH875 (A297) carries a genomic antibiotic resistance island AbaR21, plasmid pRAY and a cluster containing ISAba1-sul2-CR2-strB-strA. J Antimicrob Chemother 66(8): 1928-1930.

14. Seputiene V, Povilonis J, Suziedeliene E (2012) Novel variants of AbaR resistance islands with a common backbone in Acinetobacter baumannii isolates of European clone II. Antimicrob Agents Chemother 56(4): 1969-1973.

15. Nigro SJ, Hall RM (2011) GIsul2, a genomic island carrying the sul2 sulphonamide resistance gene and the small mobile element $C R 2$ found in the Enterobacter cloacae subspecies cloacae type strain ATCC 13047 from 1890, Shigella flexneri ATCC 700930 from 1954 and Acinetobacter baumannii ATCC 17978 from 1951. J Antimicrob Chemother 66(9): 2175-2176.

16. Nigro SJ, Hall RM (2012) Tn6167, an antibiotic resistance island in an Australian carbapenem-resistant Acinetobacter baumannii GC2, ST92 isolate. J Antimicrob Chemother 67(6): 1342-1346.

17. Toleman MA, Walsh TR (2011) Combinatorial events of insertion sequences and ICE in Gram-negative bacteria. FEMS Microbiol Rev 35(5): 912-935.

18. Nigro SJ, Farrugia DN, Paulsen IT, Hall RM (2013) A novel family of genomic resistance islands, AbGRI2, contributing to aminoglycoside resistance in Acinetobacter baumannii isolates belonging to global clone 2. J Antimicrob Chemother 68(3): 554-557.

19. Vilacoba E, Almuzara M, Gulone L, Traglia GM, Figueroa SA, et al. (2013) Emergence and spread of plasmid-borne tet(B)::ISCR2 in minocycline-resistant Acinetobacter baumannii Isolates. Antimicrob Agents Chemother 57(1): 651-654.

20. Magiorakos AP, Srinivasan A, Carey RB, Carmeli Y, Falagas ME, et al. (2012) Multidrug-resistant, extensively drug-resistant and pandrugresistant bacteria: an international expert proposal for interim standard definitions for acquired resistance. Clinical Microbiology and Infection 18(3): 268-281.

21. He S, Hickman AB, Varani AM, Siguier P, Chandler M, et al. (2015) Insertion Sequence IS26 reorganizes plasmids in clinically isolated multidrug-resistant bacteria by replicative transposition. Mbio 6(3): e00762. 